\title{
Effect of dietary protein supplementation on gene expression in the lung of lactating rats infected with Nippostrongylus brasiliensis
}

\author{
A. Masuda $^{1}$, J.E. Allen ${ }^{2}$, J.G.M. Houdijk ${ }^{1}$ and S. Athanasiadou ${ }^{1}$ \\ ${ }^{1}$ Scotland's Rural College, West Mains Road, Edinburgh, EH9 3JG, UK and ${ }^{2}$ Institution of Infection and Immunology, \\ University of Edinburgh, West Mains Road, Edinburgh, EH9 3JT, UK
}

Previous studies have shown that the breakdown of immunity to Nippostrongylus brasiliensis $(\mathrm{Nb})$, a rat nematode used as a model for gastrointestinal parasitism, is sensitive to dietary protein supply, as measured at the small intestine, the parasite niche ${ }^{(1,2)}$. Nb however goes through systemic migration, entering host's skin and migrating to lung parenchyma through blood vessels before reaching the intestine. Here we investigated for the first time the effect of dietary protein supply on host's lung in the Nb re-infected rat model.

Nulliparous female Sprague-Dawley rats were given primary infection of $1600 \mathrm{Nb} 3^{\text {rd }}$ stage infective larvae (L3) subcutaneously 14 days prior to mating. Ten days post mating confirmation, rats were offered ad libitum access to either a High Protein (H) or a Low Protein (L) diet until sampling point. Half of these animals were administered with a secondary 1600 L3 on day 2 post parturition (pp) defined as $\mathrm{Nb}+\mathrm{Nb}$ group ( $\mathrm{L} n=9, \mathrm{H} n=7)$, whereas the other half were injected with PBS, defined as $\mathrm{Nb}+\mathrm{PBS}$ group $(\mathrm{L}$ and $\mathrm{H} n=6)$. Animals that did not receive primary and secondary infections were placed as a naïve control $(\mathrm{L}$ and $\mathrm{H} n=3)$. Expression of genes related to lung inflammation, particularly alternatively activated macrophages (AAMs) including, arginase 1 (Arg1), resistin-like molecules $\alpha$ (Retnla), and matrix metalloproteinase MMP-12 (Mmp12), and a marker for classically activated macrophages (CAMs), inducible nitric oxide synthase (Nos2), was measured in the lung on day 5 pp. Data were analysed by 2 (diets) $\times$ 3 (infection status) ANOVA.

Retnla expression was significantly higher in $\mathrm{H}$ compared to $\mathrm{L}$ animals following secondary infection $(P<0.001)$, and $\mathrm{Mmp} 12$ expression tended to be higher in $\mathrm{H}$ animals $(P=0.059)$. Dietary protein did not have significant effect on Arg1 and Nos2 expression. Expression of Retnla and Mmp12 were significantly up-regulated following secondary infection $(P<0 \cdot 001)$. Argl tended to be higher $(P=0.076)$ following secondary infection whereas Nos2 expression was significantly down regulated $(P<0.001)$ after secondary infection.
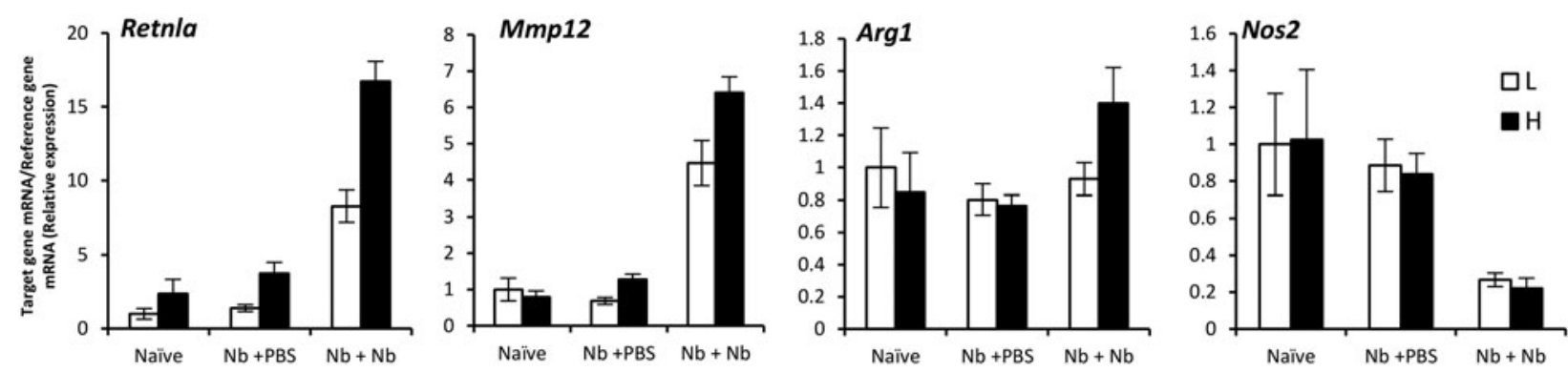

Fig. 1. Lung gene expressions on day 5 pp.

Despite the fact that the intestine is a critical site for immune response development, studies in mice have shown that the lung phase plays a crucial role in generating immunity against $\mathrm{Nb}$ re-infection ${ }^{(3,4)}$. Results presented here indicate for the first time that nutrient supplementation can affect immune responses during the lung phase of $\mathrm{Nb}$ infection. Hence, previously observed benefits of dietary protein supplementation ${ }^{(1,2)}$ may already be generated during the lung phase of the infection.

1. Houdijk JG, Jessop NS, Knox DP et al. (2005) Brit J Nutr 93, 493-499.

2. Jones LA, Houdijk JGM, Knox DP et al. (2009) Parasite Immunol 31, 412-421.

3. Harvie M, Camberis M, Tang SC et al. (2010) Infect Immun 78, 3753-3762.

4. Chen F, Liu Z, Wu W et al. (2012) Nat Med 18, 260-266. 